\title{
Data Contribution Summaries for Patient Engagement in Multi-Device Health Monitoring Research
}

\author{
Jay Rainey \\ jay.rainey@newcastle.ac.uk \\ Open Lab, Newcastle University \\ Newcastle upon Tyne, UK \\ Johanna Graeber \\ jg@allgemeinmedizin.uni-kiel.de \\ Department of General Medicine, Kiel \\ University \\ Kiel, Germany \\ Viana Zhang \\ n.zhang10@newcastle.ac.uk \\ Open Lab, Newcastle University \\ Newcastle upon Tyne, UK
}

\author{
David Verweij \\ david.verweij@newcastle.ac.uk \\ Open Lab, Newcastle University \\ Newcastle upon Tyne, UK \\ Farzaneh Farhadi \\ f.farhadi2@newcastle.ac.uk \\ School of Engineering, Newcastle \\ University \\ Newcastle upon Tyne, UK \\ Christopher N. Bull \\ christopher.bull@newcastle.ac.uk \\ Open Lab, Newcastle University \\ Newcastle upon Tyne, UK
}

\author{
Colin Dodds \\ colin.bone-dodds@newcastle.ac.uk \\ Open Lab, Newcastle University \\ Newcastle upon Tyne, UK \\ Ridita Ali \\ r.ali-602@kent.ac.uk \\ School of Computing, University of \\ Kent \\ Kent, UK \\ Jan David Smeddinck \\ jan.smeddinck@newcastle.ac.uk \\ Open Lab, Newcastle University \\ Newcastle upon Tyne, UK
}

\begin{abstract}
The rapid growth in the range of data measures from wearable and stationary sensing devices has led to the adoption of multiple devices in health research. Such multi-device setups present challenges in sustaining patient engagement to capture continuous and high-quality datasets. One approach is to present health data to patients throughout the study but often occurs upon study completion. We report on preliminary insights from a feasibility study (IDEA-FAST) where multiple devices were used by 141 patients in their free-living environments. Interviews with a subset of patients and clinicians highlight challenges and opportunities around participation, data use and interpretation, including understanding compliance and data explainability with patients. We propose that summarising metadata from device usage could foster engagement and scale across a range of technologies regardless of the specific measures or post-processing algorithms provided by devices.
\end{abstract}

\section{CCS CONCEPTS}

- Human-centered computing $\rightarrow$ Empirical studies in ubiquitous and mobile computing.

\section{KEYWORDS}

digital health, human-centered design, in-the-wild studies, wearables, sensing devices, patient engagement, human-data interaction

Permission to make digital or hard copies of all or part of this work for personal or classroom use is granted without fee provided that copies are not made or distributed for profit or commercial advantage and that copies bear this notice and the full citation on the first page. Copyrights for components of this work owned by others than the author(s) must be honored. Abstracting with credit is permitted. To copy otherwise, or republish, to post on servers or to redistribute to lists, requires prior specific permission and/or a fee. Request permissions from permissions@acm.org.

UbiComp-ISWC '21 Adjunct, September 21-26, 2021, Virtual, USA

(c) 2021 Copyright held by the owner/author(s). Publication rights licensed to ACM ACM ISBN 978-1-4503-8461-2/21/09 ..\$15.00

https://doi.org/10.1145/3460418.3479371
ACM Reference Format:

Jay Rainey, David Verweij, Colin Dodds, Johanna Graeber, Farzaneh Farhadi, Ridita Ali, Viana Zhang, Christopher N. Bull, and Jan David Smeddinck. 2021. Data Contribution Summaries for Patient Engagement in Multi-Device Health Monitoring Research. In Adjunct Proceedings of the 2021 ACM International foint Conference on Pervasive and Ubiquitous Computing and Proceedings of the 2021 ACM International Symposium on Wearable Computers (UbiComp-ISWC '21 Adjunct), September 21-26, 2021, Virtual, USA. ACM, New York, NY, USA, 6 pages. https://doi.org/10.1145/3460418.3479371

\section{INTRODUCTION}

Sensing devices are revolutionising healthcare as they offer unobtrusive ways to record continuous streams of patients' vital and behavioural signs in free-living environments, such as heart rate, brain activity, and physical movement [19,29]. This is applied across a broad range of use cases, e.g. in the assessment or monitoring of Parkinson's disease symptoms [10, 27], bipolar disorder [13], or sleep assessment $[2,28]$. Increasingly, clinical studies are being conducted where multiple sensing devices are used to generate large, multi-modal health datasets that can be used to provide a more refined insight into a range of disease symptoms [16, 21, 23]. The growing numbers of simultaneously used sensing technologies adds additional protocol requirements for patients to engage with that can impact compliance and desires to participate.

One approach to foster engagement is to provide processed data generated by these sensing devices to patients. Recent work has shown how patients feel motivated, rewarded, and included in research studies when they receive personalised reports [3], and that daily feedback on an individual's disease created positive effects on people living with Parkinson's disease [27]. Across this work, sensing data is often analysed to create health summaries that can inform personalised, data-driven consultations between clinicians and patients $[18,20]$. During such consultations, patients can be asked to contextualise the data to assist the discussion. However, this introduces additional time and effort requirements for both parties [22]. Post-processed data is commonly presented to patients 
weekly (e.g., [3, 5, 27]) or upon study completion [2, 28]. Yet, studies exclusively using end-of-participation health reports miss the opportunity to motivate patients and contextualise their data regularly whilst research participation is ongoing - although engagement is key for large-scale and longitudinal research.

Building on these challenges, we present findings based on a feasibility study (FS) that is conducted in preparation for a clinical observation study in the context of the IDEA-FAST project. Semistructured interviews were held with 24 patients and 9 clinicians to understand their experiences on-boarding, supporting, and using multiple sensing technologies, and challenges with keeping patients engaged during the study. A formative qualitative analysis of these interviews is presented with a focused reflection on the use of health data summaries and how data from sensing devices could sustain and engage patients.

Our findings contribute to future planning of multi-device research projects in digital health and are also of relevance to practical developments in a world of increasingly ubiquitous sensing that hinges on the successful integration of numerous sensing devices.

\section{MULTI-DEVICE HEALTH MONITORING}

Using multiple sensing devices to provide insights into health conditions is becoming more common with several recent and ongoing projects exploring the use of multi-device sensing for digital health. For example, $R A D A R-C N S$ uses commercially available devices to study central nervous system disease progression [21, 23], Mobilise$D$ uses smartphones and sensors to examine people's mobility [25], and the SPHERE project uses a multi-sensor and multi-modal platform (environmental, video, and wearable sensors) to passively detect health-related behaviours at home [8]. These projects use multiple devices in-the-wild, which can increase the burden for research participants and creates opportunities to explore new ways to sustain engagement. This is exemplified by a recent workshop aiming to address the user experience for multi-device ecosystems rather than the typically researched cross-device interaction techniques [30].

\section{IDEA-FAST}

Fatigue and sleep disturbances are symptoms of several chronic diseases associated with poor quality of life and increased healthcare costs [16]. A large part of measuring patient's health is using self-reported methods that can be prone to recall bias or require extensive effort to complete, e.g., sleep diaries [16]. Building on these challenges, IDEA-FAST is a five and a half year project that aims to determine "digital endpoints" or digital biomarkers through using multiple sensing devices "in-the-wild" to assess fatigue, sleep, and activities of daily living in patients with neurodegenerative diseases (e.g. Huntington's disease) and immune-mediated inflammatory diseases (e.g. rheumatoid arthritis) [16].

IDEA-FAST is composed of three stages: a pre-feasibility process where multiple sensing devices were tested by experts and used with convenient subjects to select devices for the subsequent stage as detailed in [15]; a feasibility study (FS) where eleven sensing devices were used "in-the-wild" by patients across six disease affliction groups; and a large-scale longitudinal study clinical observation study (COS) where a subset of devices from the FS will be used.
The FS aims to identify candidate digital parameters of fatigue and sleep disturbances to be examined in the larger COS. Patients were recruited across four EU and UK study centres and asked to report sleep disturbances and fatigue through surveys, questionnaires, and a digital diary during a four to five week study period [14]. Alongside this, patients are asked to use multiple sensing devices concurrently for four periods of five consecutive days. These devices measured: physical activity (e.g., [9, 11, 31]), physiology (e.g., VitalPatch [2]), EEG (e.g., [1], cognition (e.g. [6]), and socialisation parameters (via a smartphone app). Data collected from these sensing technologies will be compared with traditional clinical measures and patient-reported outcomes to understand which digital measures best correlate, and thus which devices should be used in the COS [14].

During the FS, clinicians were responsible for on-boarding and supporting patients, including monitoring device usage, and transferring data from devices. Patients were optionally provided with summary data reports generated from device provider's digital platforms (if available) upon completion of a device-use period. As human-computer interaction (HCI) researchers our focus during the FS was to understand the usability, user experience, and acceptability of these sensing devices to inform device selection suggestions, as well as the development of potential support technologies for the COS. In this paper, we present insights gained from preliminary qualitative analysis that examines the perspectives of patients and clinicians on device usage and data generated from them that could help to sustain patient engagement in multi-device studies.

\section{STUDY DESIGN}

Semi-structured interviews were conducted with patients and clinicians following their participation in the IDEA-FAST FS. These interviews aimed to understand the contextual challenges of onboarding, supporting, and keeping patients engaged during the study, and their experiences with multiple sensing devices.

Out of 24 interviewed patients, 13 were considered healthy (control), eight Parkinson's disease (PD), two inflammatory bowel disease (IBD), and one Huntington's disease (HD); more interviews are being conducted at the time of writing. Interviews lasted 49-minutes on average $(\mathrm{SD}=19 \mathrm{~m}, \mathrm{~min}=21 \mathrm{~m}, \max =92 \mathrm{~m})$ and were conducted within four weeks of patients' participation. Two researchers from one study site conducted all included patient interviews with $75 \%$ being held over videoconferencing software or telephone, and the remaining in person. Additionally, nine clinicians from four study sites were interviewed towards the end of the FS that lasted 38minutes on average $(\mathrm{SD}=09 \mathrm{~m}, \mathrm{~min}=26 \mathrm{~m}, \max =50 \mathrm{~m})$. All patient interviews were conducted in their native languages (i.e., German, English and Dutch), while clinician interviews were conducted in English. All interviews were audio recorded, transcribed, and translated into English if necessary using an automated translation service with spot-checking for translation accuracy as appropriate.

A preliminary qualitative analysis is being conducted by two authors across these datasets. The qualitative analysis of interview data was focused on understanding the challenges of multi-device use as prescribed by the existing study design protocol for both patients (for protocol engagement and device use) and clinicians (for device setup and monitoring of patient engagement), with the 
aim of informing technology design to sustain patient engagement. Labels are used to indicate individual patients $(\mathrm{P})$ and clinicians $(\mathrm{C})$.

\subsection{Ethics}

IDEA-FAST's FS study protocol [14] was registered at the German Clinical Trial Registry (DRKS00021693) and approved by the ethics committee of the Medical Faculty of Kiel University (D 491/20). Patient interviews are pseudonymous for storage and only accessible by data analysts within the consortium. Ethical approval for the clinician (anonymised) interviews reported in this paper (which was outside the scope of the FS protocol) was granted by Newcastle University (7421/2020). Verbal consent was taken as interviews were primarily held over videoconferencing software or telephone.

\section{PRELIMINARY FINDINGS}

This section outlines preliminary findings focused on the use, interpretation, and experience with health data summaries provided to patients. It examines how data from sensing devices could be used to sustain and engage patients doing longitudinal multi-device studies, and the implications for clinical practice.

\subsection{Reflecting on Health Data Summaries}

Patients used multiple sensing devices over four periods of five days each where they received daily feedback from some devices, a report following their participation, or no feedback at all, depending on the device. One of the sensing devices that monitored EEG during sleep had an associated smartphone application which did provide a report after each night. Some patients described that they were unable to engage with much of these metrics (e.g., sleep stages), as it was often unclear what the data meant, or data reports were unavailable in the provided application as data had not yet been automatically transferred. For other patients, viewing this data was used as a feedback mechanism to understand if the device was configured and recording data correctly as described by one patient:

"It was nice to see how it was going, because with [the app] I could see if it was working and if [...] the [device] battery died or whatever, it was good to keep up with that. So I knew I was doing it right." (P1)

In this way, the availability of frequent data summaries enabled patients to feel confident that they were adhering to study protocol when they "should". This meant that while some patients deviated from the agreed upon study protocol, they instead 'made up' the difference in skipped device wear days by extending their original use period. While this allows flexibility in study protocol and more convenient participation for patients, feeding back those deviations is critical to understand the overall accuracy of the dataset.

Conversely, some participants requested to discuss and clarify the data with clinicians that were provided by smartphone applications or summary health reports. Clinicians highlighted that collating reports across multiple devices was tedious, and often required collaboration with patients as some devices provided reports via apps on patient's smartphones. Clinicians reported that patients were often interested in sleep-related data so that they could observe and correlate for themselves any discrepancies between their disease conditions and the data. This required clinicians to interpret data and explain concepts (e.g. REM) to help with understanding the data. Access and availability of data was highlighted as a limiting factor in how insights could be shared to patients:

"[Patients] always want to know and have data, which is then a bit sad when we have to tell them that the sleeping reports are not available [...] we have to tell them we can't because it is all raw data." (C1)

Discussion around these health reports was seen as a key factor for motivating participation according to clinicians. It was argued that patients' access to their personalised data is required so that they can answer personal questions they have about their health:

"If patients are to be motivated to wear the devices, then they themselves must also benefit from it by getting an answer to questions they have." (C2)

Across these interviews, patients reflected how they enjoyed seeing the summary health data reports provided by clinicians after they took part in IDEA-FAST. Some patients attributed these reports as motivation for taking part, while others were not interested in the data unless they experienced poor health, e.g., did not sleep well or felt poorly. When presented with a data summary, one patient described that data was missing for some days when they thought that they had worn the device:

"The 2nd time the [report] showed that I would not have worn [device] many times, which was not true.

That irritated me a bit as I thought I worn it." (P2)

The health summary report data provided to this patient labelled missing data as "not worn" and highlighted those days in red. Upon receiving this report, the patient felt "accused" as the missing data did not match with when they had worn the device. Even though some devices provided health summary reports following study participation or in near real-time through smartphone applications, most did not. This was primarily because data transfer occurs offline (manually) after a usage period. Some patients reported feeling anxious with what the sensing devices were recording, and if it was recording data at all. This outlines a limitation with devices where near real-time feedback on usage is not currently provided whilst highlighting the benefits when it is provided.

\section{DHAPP: SUMMARISING DEVICE DATA}

Central to IDEA-FAST is the process of consistently and continuously collecting sensing data in-the-wild from patients to ensure the technologies are used during intended days and for extended periods. High quality data is required for effective analysis and to create a greater benefit to the research community and patients involved. Our preliminary findings support recent calls for increased access of health data for patients [24], and additionally suggests that not only do patients use this to reflect on indications pertaining to their health and wellbeing, but also to assess their participation in, and contributions to, a study, as well as the functionality of the device(s) in use. Across these findings, the quantity of sensing devices, companion applications, and distinct ways that health data summaries were represented increased the time and effort to participate for both patients and clinicians.

In response, and to explore ways to tackle this challenge, we have designed and developed a mobile application prototype, the Device Hub APPlication (DHAPP) that unifies how patients can monitor 


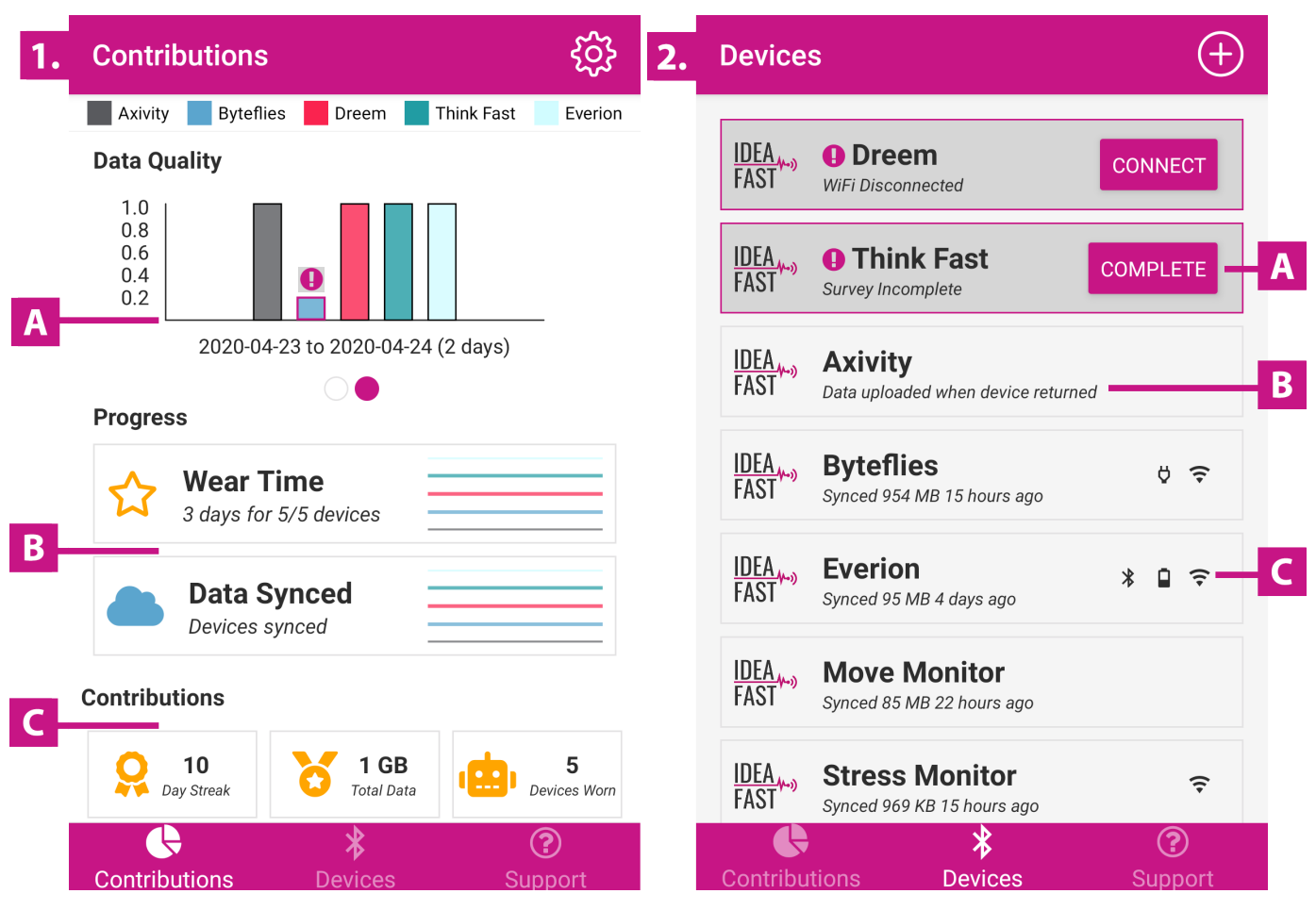

Figure 1: Exploratory designs of the Device Hub APPlication (DHAPP) that: (1) summarises data contributions from multiple sensing devices; and (2) provides a status overview of data transfer and connectivity of all technologies. The contributions screen provides feedback on data quality (1.A), progress against intended wear periods (1.B) and an aggregation of data contributions (1.C). The devices screen shows potential errors with technologies, e.g., that a daily cognition task is incomplete (2.A), when data was uploaded from a technology (2.B), and its connectivity status (2.C).

the usage of multiple sensing device during ongoing studies, which is available on GitHub [17]. Prior work highlights how patients feel motivated when they receive data, which often occurs at the end of a study period. The DHAPP summarises metadata from device usage that assumes regular access to (or control over) the raw data. This approach scales across multiple devices regardless of the specific measures provided by devices and the post-processing algorithms that are typically performed to create health reports.

Currently, DHAPP has four design features to assist patients in understanding device use, operation, and engagement with the research protocol: (1) data volume that provides a summary of participation over time; (2) data quality that notifies patients if data contributed falls below a threshold (Figure 1.1.A); (3) data transfer and connectivity that provides a status overview of all technologies in use (Figure 1.2.A-C); and (4) access to training resources for each device, i.e., documentation. This initial design explores playful gamified approaches to further engage patients at an individual level, such as streaks [7, 26]. However, our findings highlight that consideration is needed when representing 'missing' data so that patients are motivated to take action to contextualise why this occurs to reduce misunderstandings in the reported data.

\section{SUSTAINING PATIENT ENGAGEMENT}

Research studies are increasingly using multiple sensing devices to provide objective measures of a range of disease symptoms, e.g.,
$[8,23,25]$. Such multi-device health monitoring research creates barriers for participants due to the required configurations and adherence to use multiple devices. This paper has outlined preliminary qualitative findings from an ongoing multi-device study and highlights desires from patients to have (near) real-time access to data from such devices to support their study protocol compliance efforts. Building on these findings, we propose that summarising metadata from device usage is independent of sensing devices, its data, or derived health metrics, and offer a design solution for this as realised through the DHAPP.

The recognition for patients to have access to their own data [24] raises tensions around the responsibility of researchers during such long-term studies and how data can be meaningfully represented to patients during their participation in the study. A recent review of personal informatics literature suggests a need to address barriers to creating action from data captured [12]. In multi-device health monitoring studies action could take the form of participants monitoring that data is actively being contributed. Combining meaningful information across multiple data streams remains challenging. Our proposed concept of data contribution summaries help address this through an intermediary layer between raw and post-processed data that could enhance engagement. 
The workshop [4] offers an opportunity to discuss ongoing challenges that we have experienced in supporting multi-device research, how our data contribution summaries concept could be extended to other contexts, and to understand how other approaches have been applied to engage patients through data.

\section{ACKNOWLEDGMENTS}

The IDEA-FAST project has received funding from the Innovative Medicines Initiative 2 Joint Undertaking (JU) under grant agreement No 853981. The JU receives support from the European Union's Horizon 2020 research and innovation programme, EFPIA, and from Parkinson's Disease Society of the United Kingdom.

\section{REFERENCES}

[1] Pierrick J Arnal, Valentin Thorey, Eden Debellemaniere, Michael E Ballard, Albert Bou Hernandez, Antoine Guillot, Hugo Jourde, Mason Harris, Mathias Guillard, Pascal Van Beers, Mounir Chennaoui, and Fabien Sauvet. 2020. The Dreem Headband compared to polysomnography for electroencephalographic signal acquisition and sleep staging. Sleep 43, 11 (2020), 1-13. https://doi.org/10.1093/ sleep/zsaa097

[2] Yang Bai, Yu Guan, and Wan-Fai Ng. 2020. Fatigue assessment using ECG and actigraphy sensors. In Proceedings of the 2020 International Symposium on Wearable Computers (New York, NY, USA, 2020-09-12) (ISWC'20). Association for Computing Machinery, New York, NY, USA, 12-16. https://doi.org/10.1145/ 3410531.3414308

[3] Diogo Branco, Raquel Bouça, Joaquim Ferreira, and Tiago Guerreiro. 2019. Designing Free-Living Reports for Parkinson's Disease. In Extended Abstracts of the 2019 CHI Conference on Human Factors in Computing Systems (Glasgow, Scotland Uk) (CHI EA '19). Association for Computing Machinery, New York, NY, USA, 1-6. https://doi.org/10.1145/3290607.3313032

[4] Diogo Branco, Patrick Carrington, Silvia Del Din, Afsaneh Doryab, Hristijan Gjoreski, Tiago Guerreiro, Roisin McNaney, Kyle Montague, Alisha Pradhan, André Rodrigues, and Julio Vega. 2021. Wild by Design: Workshop on Designing Ubiquitous Health Monitoring Technologies for Challenging Environments. In UbiComp '21: ACM International foint Conference on Pervasive and Ubiquitous Computing (online). online, New York, NY, USA, 1-3.

[5] Diogo Branco, Ricardo Pereira, Raquel Bouça, Joaquim Ferreira, César Mendes, André Rodrigues, Kyle Montague, and Tiago Guerreiro. 2019. DataPark: A DataDriven Platform for Parkinson's Disease Monitoring. In WISH 2019 - Workgroup on Interact Systems in Healthcare Symposium (Glasgow, Scotland Uk). online, New York, NY, USA, 1-6.

[6] Francesca Cormack, Maggie McCue, Nick Taptiklis, Caroline Skirrow, Emilie Glazer, Elli Panagopoulos, Tempest A van Schaik, Ben Fehnert, James King, and Jennifer H Barnett. 2019. Wearable Technology for High-Frequency Cognitive and Mood Assessment in Major Depressive Disorder: Longitudinal Observational Study. FMIR Mental Health 6, 11 (Nov. 2019), e12814. https://doi.org/10.2196/12814

[7] Sebastian Deterding, Dan Dixon, Rilla Khaled, and Lennart Nacke. 2011. From Game Design Elements to Gamefulness: Defining "Gamification". In Proceedings of the 15th International Academic MindTrek Conference: Envisioning Future Media Environments (Tampere, Finland) (MindTrek '11). Association for Computing Machinery, New York, NY, USA, 9-15. https://doi.org/10.1145/2181037.2181040

[8] Tom Diethe, Mike Holmes, Meelis Kull, Miquel Perello Nieto, Kacper Sokol, Hao Song, Emma Tonkin, Niall Twomey, and Peter Flach. 2018. Releasing EHealth Analytics into the Wild: Lessons Learnt from the SPHERE Project. In Proceedings of the 24th ACM SIGKDD International Conference on Knowledge Discovery \& Data Mining (London, United Kingdom) (KDD '18). Association for Computing Machinery, New York, NY, USA, 243-252. https://doi.org/10.1145/3219819.3219883

[9] Silvia Del Din, Morad Elshehabi, Brook Galna, Markus A. Hobert, Elke Warmerdam, Ulrike Suenkel, Kathrin Brockmann, Florian Metzger, Clint Hansen, Daniela Berg, Lynn Rochester, and Walter Maetzler. 2019. Gait analysis with wearables predicts conversion to Parkinson disease. Annals of Neurology 86, 3 (July 2019), 357-367. https://doi.org/10.1002/ana.25548

[10] Silvia Del Din, Alan Godfrey, Claudia Mazzà, Sue Lord, and Lynn Rochester. 2016 Free-living monitoring of Parkinson's disease: Lessons from the field. Movement Disorders 31, 9 (July 2016), 1293-1313. https://doi.org/10.1002/mds.26718

[11] Aiden Doherty, Dan Jackson, Nils Hammerla, Thomas Plötz, Patrick Olivier, Malcolm H. Granat, Tom White, Vincent T. van Hees, Michael I. Trenell, Christoper G. Owen, Stephen J. Preece, Rob Gillions, Simon Sheard, Tim Peakman, Soren Brage, and Nicholas J. Wareham. 2017. Large Scale Population Assessment of Physical Activity Using Wrist Worn Accelerometers: The UK Biobank Study. PLOS ONE 12, 2 (02 2017), 1-14. https://doi.org/10.1371/journal.pone.0169649
[12] Daniel A. Epstein, Clara Caldeira, Mayara Costa Figueiredo, Xi Lu, Lucas M. Silva, Lucretia Williams, Jong Ho Lee, Qingyang Li, Simran Ahuja, Qiuer Chen, Payam Dowlatyari, Craig Hilby, Sazeda Sultana, Elizabeth V. Eikey, and Yunan Chen. 2020. Mapping and Taking Stock of the Personal Informatics Literature. Proc. ACM Interact. Mob. Wearable Ubiquitous Technol. 4, 4, Article 126 (Dec. 2020), 38 pages. https://doi.org/10.1145/3432231

[13] Pegah Hafiz, Kamilla Woznica Miskowiak, Alban Maxhuni, Lars Vedel Kessing, and Jakob Eyvind Bardram. 2020. Wearable Computing Technology for Assessment of Cognitive Functioning of Bipolar Patients and Healthy Controls. Proc. ACM Interact. Mob. Wearable Ubiquitous Technol. 4, 4, Article 129 (Dec. 2020), 22 pages. https://doi.org/10.1145/3432219

[14] IDEA-FAST. 2021. D2.1: First study subject approvals package of the Feasibility Study (FS). Retrieved June 10, 2021 from https://idea-fast.eu/wp-content/ uploads/2021/01/IDEA-FAST_D2.1_FS-Approvals_v1.0_IMI.pdf

[15] IDEA-FAST. 2021. D3.1-Device Selection Criteria and Documents / Processes for Gathering Evidence. Retrieved June 10, 2021 from https://idea-fast.eu/wp-content/uploads/2021/01/IDEA-FAST_D3.1_ DeviceSelectionCriteriaAndDocuments v1.pdf

[16] IDEA-FAST. 2021. IDEA-FAST: Identify Digital Endpoints to Assess FAtigue, Sleep and acTivities of daily living in neurodegenerative disorders and immunemediated inflammatory diseases. Retrieved June 10, 2021 from http://www.imi. europa.eu/projects-results/project-factsheets/idea-fast

[17] IDEA-FAST. 2021. Release Device Hub Application (DHAPP) .ideafast/ideafastpatientapp. Retrieved June 10, 2021 from https://github.com/ideafast/ideafastpatientapp/releases/tag/v0.1-alpha

[18] Yoojung Kim, Eunyoung Heo, Hyunjeong Lee, Sookyoung Ji, Jueun Choi, JeongWhun Kim, Joongseek Lee, and Sooyoung Yoo. 2017. Prescribing 10,000 Steps Like Aspirin: Designing a Novel Interface for Data-Driven Medical Consultations. In Proceedings of the 2017 CHI Conference on Human Factors in Computing Systems (Denver, Colorado, USA) (CHI '17). Association for Computing Machinery, New York, NY, USA, 5787-5799. https://doi.org/10.1145/3025453.3025570

[19] Walter Maetzler and Lynn Rochester. 2015. Body-worn sensors-the brave new world of clinical measurement? Movement Disorders 30, 9 (July 2015), 1203-1205. https://doi.org/10.1002/mds.26317

[20] Helena M. Mentis, Anita Komlodi, Katrina Schrader, Michael Phipps, Ann GruberBaldini, Karen Yarbrough, and Lisa Shulman. 2017. Crafting a View of SelfTracking Data in the Clinical Visit. In Proceedings of the 2017 CHI Conference on Human Factors in Computing Systems (Denver, Colorado, USA) (CHI '17). Association for Computing Machinery, New York, NY, USA, 5800-5812. https: //doi.org/10.1145/3025453.3025589

[21] Ashley Marie Polhemus, Jan Novák, Jose Ferrao, Sara Simblett, Marta Radaelli, Patrick Locatelli, Faith Matcham, Maximilian Kerz, Janice Weyer, Patrick Burke, Vincy Huang, Marissa Fallon Dockendorf, Gergely Temesi, Til Wykes, Giancarlo Comi, Inez Myin-Germeys, Amos Folarin, Richard Dobson, Nikolay V Manyakov, Vaibhav A Narayan, and Matthew Hotopf. 2020. Human-Centered Design Strategies for Device Selection in mHealth Programs: Development of a Novel Framework and Case Study. 7MIR Mhealth Uhealth 8, 5 (7 May 2020), e16043. https://doi.org/10.2196/16043

[22] Shriti Raj, Joyce M. Lee, Ashley Garrity, and Mark W. Newman. 2019. Clinical Data in Context: Towards Sensemaking Tools for Interpreting Personal Health Data. Proc. ACM Interact. Mob. Wearable Ubiquitous Technol. 3, 1, Article 22 (March 2019), 20 pages. https://doi.org/10.1145/3314409

[23] Yatharth Ranjan, Zulqarnain Rashid, Callum Stewart, Pauline Conde, Mark Begale, Denny Verbeeck, Sebastian Boettcher, Richard Dobson, and Amos Folarin. 2019. RADAR-Base: Open Source Mobile Health Platform for Collecting, Monitoring, and Analyzing Data Using Sensors, Wearables, and Mobile Devices. FMIR Mhealth Uhealth 7, 8 (01 Aug 2019), e11734. https://doi.org/10.2196/11734

[24] Sara Riggare, Jon Stamford, and Maria Hägglund. 2021. A Long Way to Go: Patient Perspectives on Digital Health for Parkinson's Disease. Journal of Parkinson's Disease Preprint (2021), 1-6. https://doi.org/10.3233/JPD-202408 Preprint.

[25] Lynn Rochester, Claudia Mazzà, Arne Mueller, Brian Caulfield, Marie McCarthy, Clemens Becker, Ram Miller, Paolo Piraino, Marco Viceconti, Wilhelmus P Dartee, et al. 2020. A roadmap to inform development, validation and approval of digital mobility outcomes: the Mobilise-D approach. Digital Biomarkers 4, 1 (2020), 13-27.

[26] Jan David Smeddinck, Marc Herrlich, Max Roll, and Malaka Rainer. 2014. Motivational Effects of a Gamified Training Analysis Interface. , 8 pages.

[27] Janet M.T. van Uem, Katrin S. Maier, Svenja Hucker, Olga Scheck, Markus A. Hobert, Ana Teresa Santos, Øyvind Fagerbakke, Frank Larsen, Joaquim J. Ferreira, and Walter Maetzler. 2016. Twelve-week sensor assessment in Parkinson's disease: Impact on quality of life. Movement Disorders 31, 9 (May 2016), 1337-1338. https://doi.org/10.1002/mds.26676

[28] Bing Zhai, Ignacio Perez-Pozuelo, Emma A. D. Clifton, Joao Palotti, and Yu Guan. 2020. Making Sense of Sleep: Multimodal Sleep Stage Classification in a Large, Diverse Population Using Movement and Cardiac Sensing. Proc. ACM Interact. Mob. Wearable Ubiquitous Technol. 4, 2, Article 67 (June 2020), 33 pages. https://doi.org/10.1145/3397325 
[29] Heng Zhang, Jan Smeddinck, Rainer Malaka, Yao Shu, Chong Chen, Bo He, Zengjun Fu, and Michael Lawo. 2019. Wireless non-invasive motion tracking of functional behavior. Pervasive and Mobile Computing 54 (March 2019), 29-44. https://doi.org/10.1016/j.pmcj.2019.01.006

[30] Ru Zhang, Yuanchun Shi, Björn Schuller, Elisabeth André, Sharon Oviatt, Aaron Quigley, Nicolai Marquardt, Ilhan Aslan, and Ran Ju. 2021. User Experience for Multi-Device Ecosystems: Challenges and Opportunities. In Extended Abstract of the 2021 CHI Conference on Human Factors in Computing Systems (Yokohama, Japan) (CHI EA '21). ACM, New York, NY, USA, Article 111, 5 pages. https: //doi.org/10.1145/3411763.3441325

[31] Tianyue Zheng, Zhe Chen, Chao Cai, Jun Luo, and Xu Zhang 2020. V2iFi: In-Vehicle Vital Sign Monitoring via Compact RF Sensing. Proc. ACM Interact. Mob. Wearable Ubiquitous Technol. 4, 2, Article 70 (June 2020), 27 pages. https: //doi.org/10.1145/3397321 